\title{
Hydrophobic surface patterning with soft, wax-infused micro-stamps
}

\author{
Soroosh Torabi ${ }^{1}$, Zhuoyun $\mathrm{Cai}^{2}$, Jonathan T. Pham², Christine A. Trinkle ${ }^{1^{*}}$
}

${ }^{1}$ Mechanical Engineering, University of Kentucky, Lexington, KY, 40506, United States

${ }^{2}$ Chemical and Materials Engineering, University of Kentucky, Lexington, KY, 40506, United States

*Corresponding author: 277 Ralph G. Anderson Building, University of Kentucky, Lexington, KY 40506-

0503, United States. Phone: 859-218-0640. E-mail:c.trinkle@uky.edu

\begin{abstract}
$\underline{\text { Abstract }}$
In this paper, we demonstrate an easy-to-use and inexpensive stamping method that can be used to generate hydrophobic paraffin surface patterns on a variety of materials and surface geometries, including flat and curved surfaces. In this method, a waxy Parafilm sheet is placed on the back of a polydimethylsiloxane (PDMS) stamp containing microscale surface features; when heated above the melting temperature, the paraffin liquefies and diffuses into the stamp, which functions as a paraffin reservoir. The directional diffusion of the paraffin allows only a thin layer of liquid to be present at the micropatterned stamp surface. Hence, when it is put into contact with a target surface, it transfers a paraffin pattern with submicron lateral resolution and sub-100 $\mathrm{nm}$ thickness. By using XPSanalysis to track paraffin diffusion process within the PDMS stamp, the diffusion coefficient in PDMS is estimated to be 5.3 $\times 10^{-11} \mathrm{~m}^{2} / \mathrm{s}$ at $65^{\circ} \mathrm{C}$. In addition to improved patterning, the paraffin-saturated PDMS stamp removes the need for re-inking for consecutive patterning. In general, our process allows for rapid deposition of complex, multi-layer paraffin patterns on a variety of surfaces.
\end{abstract}
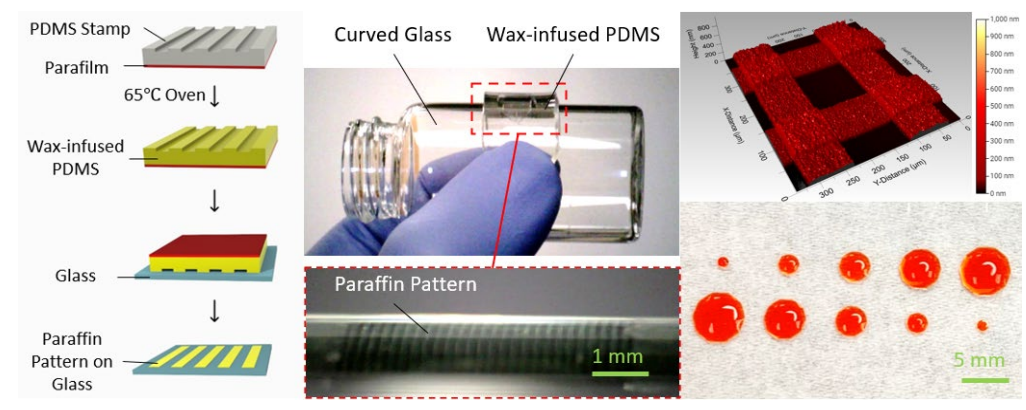

\section{$\underline{\text { Keywords }}$}

Hydrophobic patterning, Polydimethylsiloxane (PDMS) stamp, Paraffin diffusion, Microcontact printing. 


\section{Introduction}

Hydrophobic micropatterns make it possible to engineer surface wetting and adhesive properties in interesting, unique ways, which makes them useful in numerous applications. For example, incorporating selective hydrophobic and hydrophilic regions in microfluidic systems has been used to demonstrate precise control and manipulation of flow [1-3] and even the creation of individual droplets [4-6]. Hydrophobic patterning can also be used to define adhesion sitesfor patterning of proteins $[7,8]$ and cells $[9,10]$ in drug discovery and high-throughput screening applications.

It is possible to generate hydrophobic micropatterns by topographical modification of the primary surface itself-for example, by using plasma exposure $[11,12]$ or etching processes $[13,14]$ to selectively change the local surface roughness. Alternatively, micropatterns can be created by the selective addition of a secondary material. Various materials have been used in this capacity, including self-assembled monolayers (SAMS) [15, 16], biomolecules [17], and polymers [18]. Chemical bonding, lift-off, and microcontact printing have been used to deposit these materials, creating patterns with distinctive hydrophobic and hydrophilic regions.

Among the materials suitable for additive methods, paraffin wax is interesting because of its low cost, accessibility, and biocompatibility [19]; waxes are found in a host of applications, from candles and artistic sculptures to coatings for cheese and children's crayons. Additionally, its moderate melting temperature $\left(42-80^{\circ} \mathrm{C}\right.$ [20]) makes it compatible with a wide range of fabrication techniques. Carlen et al. [21, 22] depositeda 3-10 $\mu \mathrm{m}$ thick paraffin film using thermal evaporation and patternedit using a $\mathrm{CF}_{4}: \mathrm{O}_{2}$ reactive ion etching (RIE) process. Thinfilms of paraffin $(2-10 \mu \mathrm{m})$ have also been deposited using spin-coating and patterned using lift-off [23], wet etching, or dry etching [24]. While these methods are capable of micrometer-scale resolution, they require access to advanced and expensive equipment or the use of harsh chemicals. Another patterning method is paraffin inkjet printing, broadly used in paper microfluidics [25] and more recently in hydrophobic patterning of glass and plastic surfaces [19]. While this method can generate new patterns quickly - making it especially useful in prototyping applications - it also requires access to specialized equipment and is best suited for lower resolution ( $30 \mu \mathrm{m})$ patterns.

Alternatively, microcontact printing can also be used to create paraffin micropatterns; in this process, a stamp with a micropatterned surface transfers material onto a second substrate. Kuo et al. [9] used a PDMS stamp to transfer molten paraffin wax from a Parafilm sheet onto glass and aluminum surfaces. This method relies on surface tension to transfer a layer of liquid paraffin to a second surface. While microcontact printing is relatively simple and inexpensive compared to other methods, in practice, the liquid wax layer can inadvertently serve as a viscous lubricant, causing the stamp to slide when pressed onto the secondary surface or make it challenging to create thin, sub-micron layers.

In this work, we present an inexpensive stamping method that can be used to deposit hydrophobic paraffin patterns with sub-100 nm thickness on a variety of surfaces. This method relies on diffusion of paraffin through a PDMS stamp, which serves both as the patterning mechanism and as a reservoir for the paraffin (Fig. 1a). This avoids the potentially messy process of directly handling liquid paraffin, and upon contact printing, it forms an ultra-thin hydrophobic pattern. The master molds for the PDMS stamps can be fabricated using either microfabrication methods such as photolithographic patterning of SU-8 on silicon or using commercially-available 3D printing, which avoids the need for a cleanroom environment. Wax-infused patterning of paraffin enables high resolution hydrophobic patterns on multiple substrate materials, on flat or curved surfaces, and it can even be used to generate complex multi-layer pattems on the same surface (Fig. 1 b, c). The thickness of the deposited paraffin is a function of the contact time and 
can be tuned to values between $80 \mathrm{~nm}$ and $350 \mathrm{~nm}$. Additionally, because the PDMS stamp can absorb a substantial amount of paraffin, the stamps can be used numerous times without re-inking.

(a)

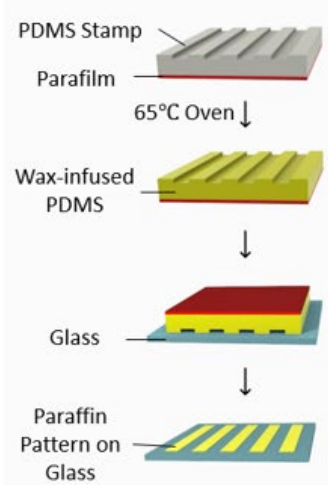

(b)

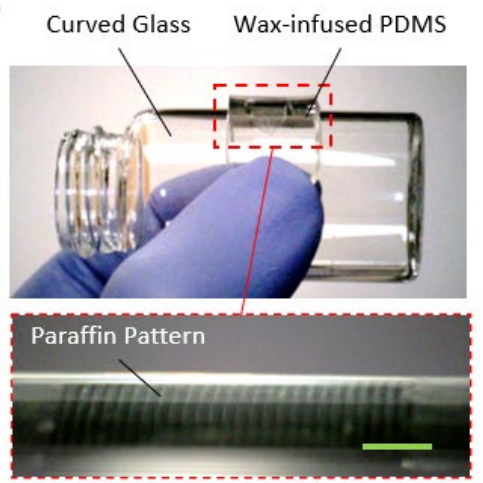

(c)

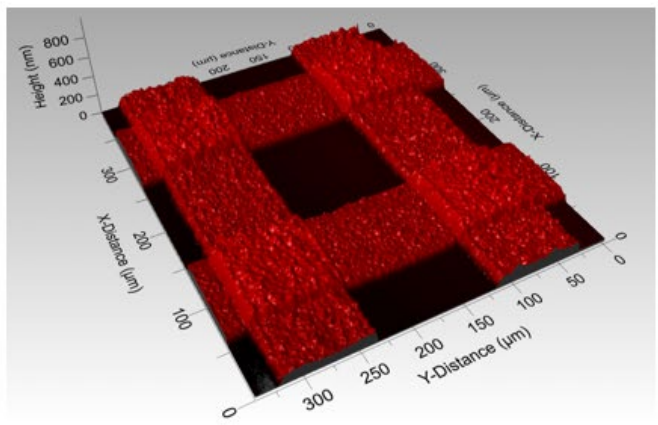

Figure 1. a) Wax-infused patterning of paraffin. Scale bar $=1 \mathrm{~mm}$; b) paraffin pattern on curved glass; c) optical profilometry scan of a multi-layer pattern created by stamping multiple times on glass. Scale bar $=1 \mathrm{~mm}$.

\section{Methods and materials}

\section{Stamp fabrication}

The master molds for the PDMS stamps were made using either 3D printing or photolithography. For 3Dprinted molds, a stereolithography-based 3D printer (Form 3, Formlabs) was used to print master molds. Following printing, the parts were thoroughly washed and sonicated in an isopropyl alcohol (IPA) bath (FormWash, Formlabs) for $10 \mathrm{~min}$. The parts were then blow dried with compressed air to remove excess IPA and allowed to air dry at room temperature for 30 minutes. The parts were then placed in a UV oven (FormCure, Formlabs) for $30 \mathrm{~min}$ at $60{ }^{\circ} \mathrm{C}$ temperature. After curing, a thin layer of mold release (Ease Release ${ }^{\circledR}$ 200, Mann Release Technologies) was sprayed on the 3D printed parts to facilitate peeling of the PDMS after curing.

For master molds made via soft lithography, one layer of SU-8 photoresist (SU-8 3050, MicroChem) was spincoated onto a clean silicon wafer ( $1500 \mathrm{rpm}$ for $30 \mathrm{~s}$ ), followed by a $1 \mathrm{~min}$ baking step on a hotplate at $65^{\circ} \mathrm{C}$, followed by a second spincoated layer of SU-8 (1000 rpm for $\left.30 \mathrm{~s}\right)$-resulting in a total thickness of $200 \mu \mathrm{m}$. Then the SU-8/silicon wafer was heated on a hotplate for $1 \mathrm{~min}$ at $65^{\circ} \mathrm{C}$, followed by $25 \mathrm{~min}$ at $95{ }^{\circ} \mathrm{C}$. The wafer was then exposed to UV through a patterned photomask and then post-exposure baked on a hotplate for $1 \mathrm{~min}$ at $65^{\circ} \mathrm{C}$ followed by $5 \mathrm{~min}$ at $95^{\circ} \mathrm{C}$. After cooling to room temperature, the uncross-linked SU-8 was removed by vigorouslyagitating the wafer for $5 \mathrm{~min}$ in SU-8 developer. The wafer was then rinsed in isopropyl alcohol (IPA 70\% in water, Sigma-Aldrich) and dried with nitrogen gas. To create a hydrophobic surface, the wafer was exposed to trichloro $(1 \mathrm{H}, 1 \mathrm{H}, 2 \mathrm{H}, 2 \mathrm{H}$-perfluorooctyl)silane (Sigma-Aldrich) under vacuum for $40 \mathrm{~min}$. Acrylic sheets (McMaster-Carr) were laser-cut and clamped to the silicon wafers in order to form sidewalls for the molds.

The PDMS stamps were made by mixing polydimethylsiloxane base and curing agent (Sylgard 184, Dow Corning) in a 10:1 ratio and degassing under vacuum for $1 \mathrm{~h}$. The degassed PDMS mixture was poured into either a 3D printed or SU-8 master mold and placed in a $65^{\circ} \mathrm{C}$ oven for $2 \mathrm{~h}$. The molds were then removed from the oven; after the assembly cooled to room temperature, the cured PDMS stamp was peeled from the mold. 


\section{Wax-infused stamping}

For wax-infused stamping, a layer of Parafilm was placed on a glass slide, and then the PDMS stamp was placed on top, with the back of the stamp - the surface opposite the patterned surface-in contact with the paraffin (Fig 1a). The assembly was then placed in a $65^{\circ} \mathrm{C}$ oven for $24 \mathrm{~h}$ to saturate the PDMS with paraffin. To transfer the wax pattern to a target surface, the stamp was removed from the oven and the patterned surface of the PDMS was placed against the target substrate for 5 seconds at room temperature. When using the saturated stamp for multiple consecutive patterns, the stamp was reheated in the $65^{\circ} \mathrm{C}$ oven for approximately 3 min after each stamping step.

\section{Paraffin diffusion characterization}

Blocks of unpatterned PDMS ( $8 \mathrm{~mm} \times 4.5 \mathrm{~mm} \times 1.5 \mathrm{~mm}$ ) were cast using methods similar to that in the Stamp Fabrication section. A layer of Parafilm was placed on a glass slide in a $65^{\circ} \mathrm{C}$ oven for $10 \mathrm{~min}$. Then, the PDMS blocks were placed on top of molten paraffin for either 20 minutes to capture transient diffusion of the paraffin into the PDMS, or for $24 \mathrm{~h}$ to create fully paraffinated PDMS. After the incubation time, the blocks were removed from the oven and allowed to cool to room temperature. X-ray Photoelectron Spectroscopy (XPS) (K-Alpha, Thermo Scientific) was used to trace atomic percentages of silicon, oxygen and carbon on the side surface of the PDMS cubes. Additionally, a plain PDMS block was analyzed to capture the PDMS XPS signal without paraffin.

\section{$\underline{\text { Results and Discussion }}$}

\section{Pattern formation, transparency, and stability}

Our wax-infused patterning method relies on the fact that liquid paraffin can freely diffuse into a solid PDMS structure. This makes it possible to saturate a PDMS stamp with paraffin and use the same stamp numerous times without re-inking. Saturating the PDMS with paraffin is performed by placing a PDMS stamp with a Parafilm backing in a $65^{\circ} \mathrm{C}$ oven - just above the melting temperature of the paraffin wax. The molten paraffin diffuses into the PDMS stamp, and it reaches the outer boundaries of the stamp and wets those interfaces. When the stamp is removed from the oven and placed on a target substrate at room temperature, the outer molten paraffin layer temperature drops below the melting point and solidifies on the surface. When the stamp is removed, the solidified paraffin pattern is retained on the target substrate. Because the paraffin is sourced only through the stamp and there is minimal molten paraffin present at the stamping interface, there is no distortion or blurring of the transferred pattem. This results in high fidelity hydrophobic patterns on the substrate that are nearly optically transparent, as shown in Fig. S1.

Due to the elasticity of the PDMS stamp, it can be bent to create conformal contact with non-flat surfaces, even when saturated with paraffin wax. To demonstrate this, we wrapped a $1.6 \mathrm{~mm}$ thick paraffinsaturated stamp around a $27.4 \mathrm{~mm}$ diameter glass vial, as shown in Fig. 1b. The resulting pattern transferred without any observed defects, indicating good contact during the transfer process.

Multi-layer paraffin patternscan al so be achieved by consecutive patterning on the same target substrate. Because of the short contact time, this method can be applied multiple times on a single substrate without disrupting the previous pattern(s). Fig 1.c shows a multi-layer paraffin pattern created by two consecutive 
stamping steps, where the stamp was rotated by $90^{\circ}$ between the first and second contact. This makes it possible to create complex paraffin geometries by layering sequential patterns of one or more PDMS stamps.

(a)

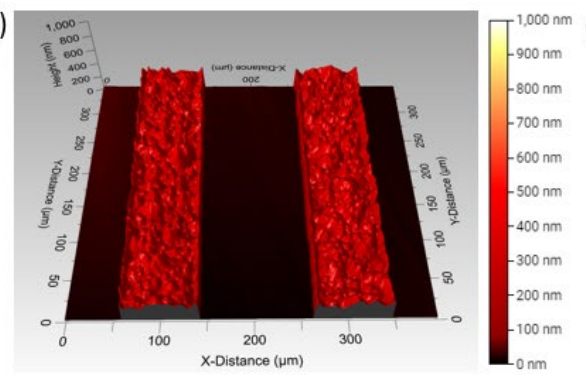

(b) 400

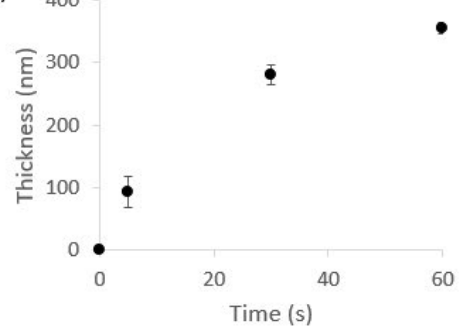

(c) 120

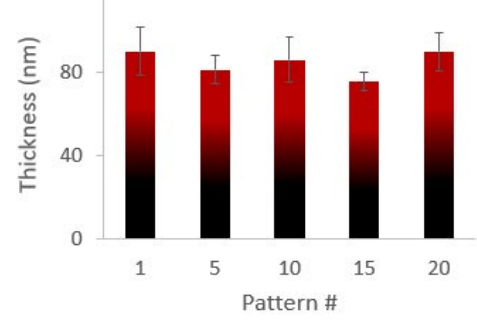

Figure 2. a) 3D topography of paraffin patterns deposited using our wax-infused stamping method; b) thickness of deposited paraffin pattern thickness vs contact time; c) thickness of deposited paraffin for 20 consecutive patterns using the same stamp.

The average thickness of the paraffin layer was $355 \mathrm{~nm} \pm 10 \mathrm{~nm}$ (mean \pm standard deviation), when the stamp and target surface were in contact for 30 seconds (Fig. 2a). By contrast, patterns created using microcontact printing for the same amount of time have been reported as being $4.5 \mu \mathrm{m}$ thick [9]. The difference in pattern thickness between the two methods implies that there is a smaller paraffin volume present on the surface of the wax-infused stamp compared to the microcontact printing stamp during contact.

The nature of the paraffin deposition makes it possible to adjust the paraffin thickness by changing the amount of time the stamp remained in contact with the target surface. As shown in Fig. $2 b$, the thickness of the deposited paraffincan be as thin as $84 \mathrm{~nm} \pm 18 \mathrm{~nm}$ for 5 seconds of contact time and up to $355 \mathrm{~nm}$ $\pm 9.6 \mathrm{~nm}$ for 60 seconds of contact. 5 seconds is the minimum practical time for manual stamping, but it may be possible to achieve thinner layers by reducing the contact time in a more controlled fashion. The ability to make controlled submicron thickness patterns could be useful in applications where very fine pattern resolution is required, and thicker layers would create fragile, high-aspect-ratio paraffin structures. Additionally, while solid paraffin is white in color and opaque, the submicron paraffin patterns here are transparent, which avoids any optical interference when imaging (Fig. S2).

The geometry of the deposited paraffin patterns matches that of the original PDMS stamp with very high fidelity. This is significant because PDMS swells with absorption of many of the liquids to which it is permeable [26-28]; however, Dangla et al [29] reported no significant swelling of PDMS upon absorption of liquid paraffin, which allows the fully infused PDMS stamp in our method to maintain its original geometry. To verify this, we imaged microscale features on the PDMS stamp before saturation with paraffin and compared it to the resulting pattern deposited using the same stamp (Fig. S1); the geometry of the two is virtually identical.

Using paraffin-saturated stamps also makes it possible to reuse the same stamp multiple times without needing to re-ink. We demonstrated this by repetitive stamping using a single PDMS stamp; the stamp was briefly reheated in between consecutive stamping steps ( $3 \mathrm{~min}$ in an $65^{\circ} \mathrm{C}$ oven) and brought into contact with a fresh, untreated glass surface each time. The resulting patterns were measured and showed no significant variation in feature height (Fig. 2c) even after 20 consecutive stamping events, demonstrating that the PDMS stamp functioned as an adequate reservoir without noticeable depletion of the paraffin wax. 
To test the stability of the paraffin micropatterns in solutions relevant to biological studies, patterned surfaces were submerged in cell culture media, or $70 \%$ ethanol at $37^{\circ} \mathrm{C}$ for 4 days. Inspection of the patterns submerged in media showed no significant loss of pattern coverage during this time, although the surface roughness of the patterns increased (Fig. S3). The results of the media tests are significant, given that paraffin wax has been used in cell culture previously $[9,19]-$ so the ability of these patterns to withstand typical incubator conditions for several days supports its use in generatingsurfacesfor selective cell adhesion and manipulation.

However, when the same paraffin patterns were exposed to static culture in an $70 \%$ ethanol solution, they degraded significantly during the 96-hour timeframe. Because the ethanol is able to successfully break up the paraffin layer, this makes it an ideal candidate for intentional removal of paraffin patterns. By rinsing the substrate with $70 \%$ ethanol and wiping off the liquid with a KimWipe, patterns can be completely removed-leaving no trace on the substrate-and a new pattern can be deposited on the same spot (Fig. S4). Patterns can be successfully changed multiple timesin this way without any detectible residue.

\section{Quantifying paraffin diffusion into PDMS stamps}

Other researchers have used absorption of molten paraffin into PDMS to control PDMS transparency [30], small molecule and gas permeability [26], and surface energy [31]; but saturation was mostly achieved by submerging the PDMS in a liquid wax bath [30-31]. This ensures absorption of paraffin into all PDMS surfaces, but a thick, non-uniform paraffin layer is formed around the block after removal from the bath. By contrast, our mechanism only places the original paraffin source into contact with the PDMS surface opposite of the patterned features. When the surrounding temperature is raised, the paraffin melts and follows a unidirectional diffusion path through the PDMS from the source side to the surface containing the micropatterned geometry.

As the paraffin diffuses along this path, it alters both the optical transparency of the PDMS and the material's carbon content, making it possible to track diffusion optically or by using XPS analysis. To measure this diffusion, we prepared a series of PDMS blocks with the same geometry ( $8 \mathrm{~mm} \times 4.5 \mathrm{~mm} \times$ $1.5 \mathrm{~mm}$ ) and incubated some of them in a $65^{\circ} \mathrm{C}$ oven with Parafilm on one surface for either 20 minutes or 24 hours. Other blocks were not exposed to any paraffin to provide a negative control. Brightfield images of the three sample types are shown in Fig. 3a, and demonstrate a clear gradient that forms after only 20 minutes of exposure to liquid paraffin. After 96 hours, the samples are uniformly opaque. 

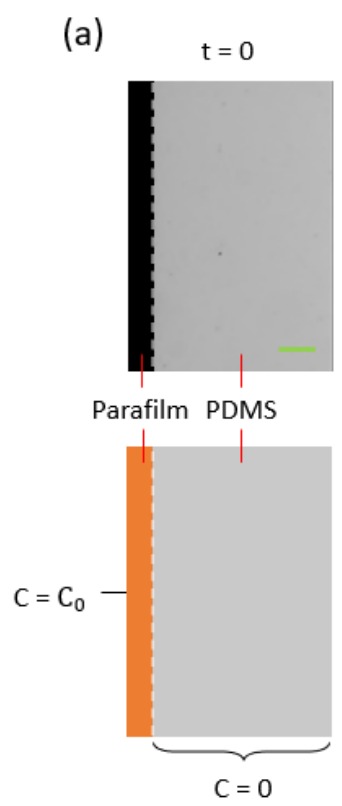

$\mathrm{t}=20 \mathrm{mins}$

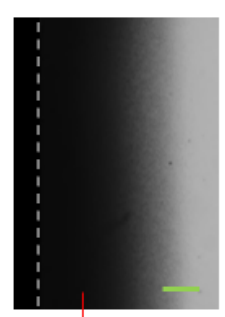

Diffused Paraffin

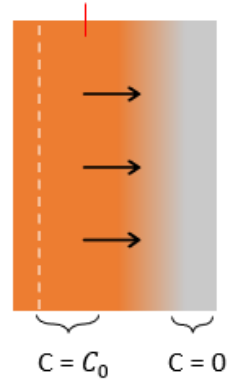

$\mathrm{t}=96 \mathrm{hrs}$
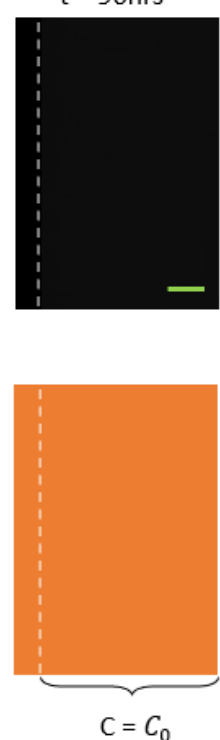

(b)

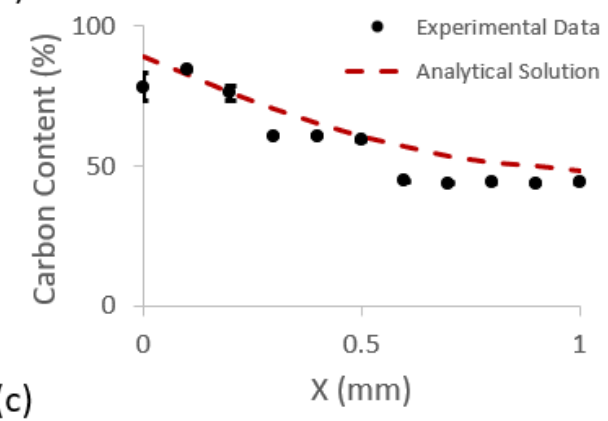

(c)

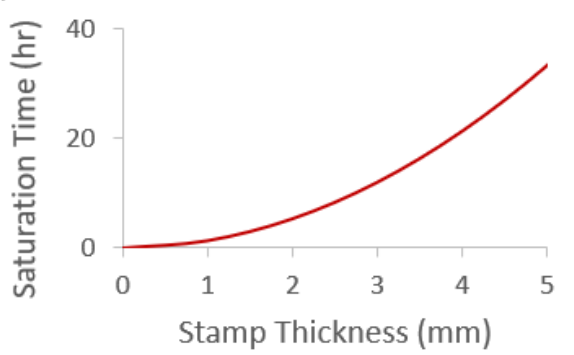

Figure 3. Paraffin diffusion in PDMS. a) Schematic view (top), and microscopic images (bottom) of the paraffin diffusion in PDMS; scale bar $=200 \mu \mathrm{m}$. b) XPS measurements of carbon content as a function of distance from the paraffin source in PDMS after 20 minutes of diffusion compared to analytical solution of 1-D diffusion where $D=5.3 e$ $11 \mathrm{~m}^{2} / \mathrm{s}$. c) Estimated saturation time for given PDMS stamp thickness based on $D=5.3 e-11 \mathrm{~m}^{2} / \mathrm{s}$.

This data qualitatively follows what is expected in 1-D diffusion from an infinite source at constant concentration, $C_{0}$. To test this in a more quantitative manner, we measured the samples using XPS at a number of discrete points at specified intervals from the source boundary. The resulting carbon content measurements, shown in Fig. 3b, show a similar gradient trend that was observed in the optical inspection. If we model this diffusion as 1-D transient diffusion from a constant-concentration source, the internal paraffin distribution $(C)$ as a function of time $(t)$ and distance from the source $(x)$ would follow the solution to Fick's second law of diffusion under these conditions:

$$
C(x, t)=C_{0} \cdot \operatorname{erfc}(x / \sqrt{D \cdot t})
$$

By using a best-fit of our data to this curve, we estimate the diffusion coefficient of paraffin in PDMS as being $5.3 \times 10^{-11} \mathrm{~m}^{2} / \mathrm{s}$.

The predicted diffusion coefficient can be used as a basis to estimate the diffusion time to fully saturate the PDMS stamps for any given thickness (Fig. 3c). This makes it possible to estimate the time necessary for a PDMS stamp to be fully inked by paraffin. For example, for the $1.5 \mathrm{~mm}$ PDMS stamps used here, the required saturation time was $\sim 5$ hours. Once the stamp is ready, each stamping deposits $\sim 84 \mathrm{~nm}$ layer of paraffin on the substrate. Based on the predicted values in Fig. $3 c$, the time required to replenish the stamp interface after each stamp is $<<1 s$, which furthervalidates the robustness of this method for rapid and consecutive patterning, as demonstrated experimentally in Fig. 2c. 


\section{Potential applications for paraffin micropatterns}

The hydrophobic nature of paraffin patterns means that they can be used to confine small liquid droplets on a surface or to guide liquid motion. One of the most appealing applications for hydrophobic patterning is droplet isolation and manipulation in drug screening [32] and cell patterning [33]. This is achieved by creating repetitive hydrophobic boundaries on a hydrophilic surface that can confine liquid droplets or cells to the hydrophilic regions. To illustrate this, we generated a 3D printed mold with circular features between 1 and $5 \mathrm{~mm}$ in diameter, cast a PDMS stamp from this mold, saturated the stamp with paraffin and used it to generate a paraffin pattern on an otherwise untreated glass surface. Paraffin formed the "background" of the pattern, leaving an array of hydrophilic circles on the glass. When aliquots of colored water with volumes ranging from 0.2 to $8 \mu \mathrm{L}$ were pipetted onto this surface, the hydrophobic pattem successfully confined the droplets to the pre-patterned regions, as shown in Fig. 4a.

(a)

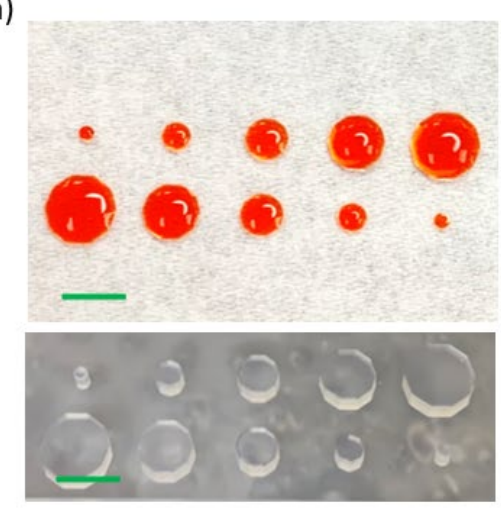

(b)

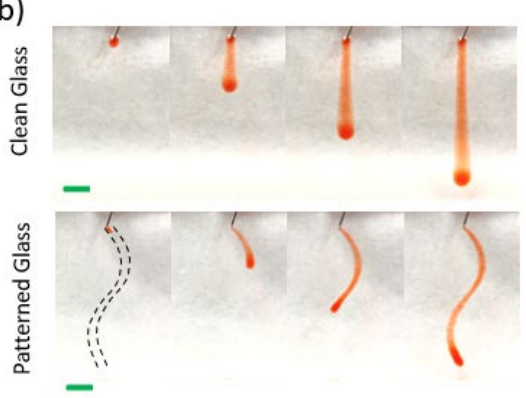

(c)

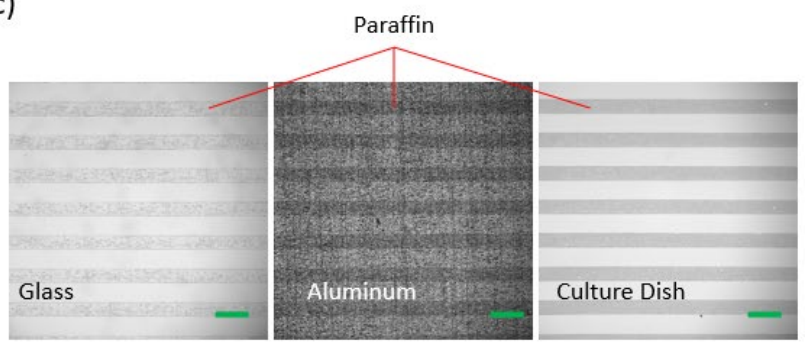

(d)

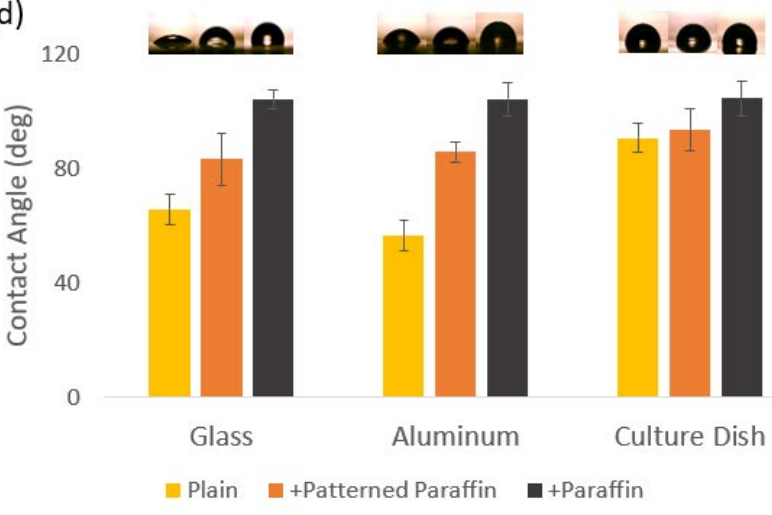

Figure 4. (a,b) Liquid confinement using paraffin patterns: a) colored water volumes of $0.2,1,3,5$, and $8 \mu$ L confined by hydrophobic paraffin circles with respective diameters of 1, 2, 3, 4, and $5 \mathrm{~mm}$ (top) vs 3D printed master mold (bottom), scale bar $=5 \mathrm{~mm}, b$ ) confined water flow on patterned glass (bottom) vs untreated glass (top), scale bar = $5 \mathrm{~mm}$. (c,d) Modified contact angle on paraffin patterned surfaces: c) striped paraffin patterns on glass, polystyrene culture dish, and aluminum surfaces, scale bar $=200 \mu \mathrm{m}$, d) contact angle of water on unpatterned surfaces, surfaces with uniform paraffin coating, and surfaces with striped paraffin pattern.

A similar method was used to guide liquid motion on a patterned surface. We created a 3D printed mold with a wavy-line feature to generate a wavy paraffin surface pattern on glass. Both the patterned glass and a clean glass slide were placed at an $80^{\circ}$ angle and colored water was deposited at a rate of 200 $\mu \mathrm{L} / \mathrm{min}$ onto the upper region of the glass, as shown in Fig. $4 \mathrm{~b}$. On the plainglass surface, the water stream moves straight down the surface, as guided by gravity, and spreads out laterally as the liquid wets the hydrophilic surface. On the wavy patterned glass, the hydrophobic edges of the pattern create a surface energy barrier and force the water to remain confined to the paraffin-free regions and maintain a constant path width. These types of hydrophobic patterns can be used to confine liquid on macroscale surfaces 
and can also be used for applications like wall-less microfluidics [33-35] where liquid flow needs to be guided on a specific track.

Paraffin wax micropatterns can be applied to numerous different materials, and these patterns can be used to directly confine liquid as shown the previous experiments, or smaller features can be used to tune the apparent contact angle of the surface. We applied a pattern of $110 \mu \mathrm{m}$ width lines to glass, al uminum, and polystyrene surfaces (Fig. 4c). Then we measured the contact angle of a $10 \mu \mathrm{L}$ water droplet placed on untreated surfaces, surfaces with the $110 \mu \mathrm{m}$ paraffin patterns, and surfaces that had been stamped with a uniform layer of paraffin. The results, shown in Fig. 4d, display a significant change in the water contact angle between plain and fully paraffin-coated substrates; the value for the fully paraffin coated surfaces is also uniform across materials, demonstrating that the deposited paraffin layer is sufficient to mask the effects of the underlying substrate on contact angle.

On all three materials, the contact angle of water on surfaces with the striped paraffin pattern lies between the value for the bare material and the fully-paraffin coated material. The apparent contact angle $\left(\theta_{c}\right)$ for a surface containing an al ternating pattern of two regions with unique contact angles $\left(\theta_{1}\right.$ and $\left.\theta_{2}\right)$ can be predicted using the Cassie-Baxter equation[36]:

$$
\cos \theta_{c}=f\left(\cos \theta_{1}-\cos \theta_{2}\right)+\cos \theta_{2}
$$

Where $f$ is the fraction of the surface area covered by the region with contact angle $\theta_{1}$. The paraffin stripes in the pattern used here had a width of $110 \mu \mathrm{m}$ and a gap of $90 \mu \mathrm{m}$ between them, resulting in a value of $f=0.55$ for all surfaces. As shown in Fig. $4 \mathrm{~d}$, the values for $\theta_{1}$ were $66^{\circ}, 57^{\circ}$, and $91^{\circ}$ for glass, aluminum, and polystyrene respectively, and $\theta_{2}$ was measured to be $104^{\circ}$ for paraffin coating on all substrates. Using the above equation, the predicted contact angle on paraffin-patterned glass, aluminum, and polystyrene surfaces $\left(87^{\circ}, 88^{\circ}\right.$, and $98^{\circ}$ respectively) matches the measured contact angle $\left(83 \pm 9^{\circ}\right.$, $86 \pm 3^{\circ}$, and $94 \pm 7^{\circ}$ respectively) rather well. Therefore, it is likely that this method can be used to generate a wide of range water contact angles on a surface by changing the dimensions on the paraffin patterns.

\section{Conclusions}

We have demonstrated that PDMS micropatterned stamps combined with an inexpensive, easy to handle paraffin wax source (Parafilm) can be used to generate high fidelity hydrophobic patterns on a variety of surface materials. Because paraffin can readily absorb into PDMS, the stamp serves as a reservoir for paraffin, so there is no need for a re-inking step after each stamping. The printed paraffin can be as thin as $85 \mathrm{~nm}$, and the pattern thickness is adjustable by changing the contact time. Furthermore, the paraffin patterns are relatively stable in cell culture media, showing promise for biological patterning applications. The PDMS master mold can be generated using traditional photolithography processes if submicron resolution is required, or lower resolution molds $(\sim 25 \mu \mathrm{m})$ can be fabricated using 3D printing, which eliminates the need for a cleanroom environment, and makes this an attractive process for low-cost, rapid generation of surface patterns.

\section{Acknowledgements}

This material is based upon work supported by the National Science Foundation under Cooperative Agreement No. 1849213. 


\section{$\underline{\text { References }}$}

1. Handique, K., et al. Microfluidic flow control using selective hydrophobic patterning. in Micromachined Devices and Components III. 1997. International Society for Optics and Photonics.

2. Ouyang, Y., et al., Rapid patterning of 'tunable'hydrophobic valves on disposable microchips by laser printer lithography. Lab on a Chip, 2013. 13(9): p. 1762-1771.

3. Dal Dosso, F., et al., Innovative hydrophobic valve allows complex liquid manipulations in a selfpowered channel-based microfluidic device. ACS sensors, 2019. 4(3): p. 694-703.

4. $\quad \mathrm{Hu}, \mathrm{H}$., S. Yu, and D. Song, No-loss transportation of water droplets by patterning a desired hydrophobic path on a superhydrophobic surface. Langmuir, 2016. 32(29): p. 7339-7345.

5. Efremov, A.N., et al., Micropatterned superhydrophobic structures for the simultaneous culture of multiple cell types and the study of cell-cell communication. Biomaterials, 2013. 34(7): p. 1757-1763.

6. Tenjimbayashi, M., et al., Droplet motion control on dynamically hydrophobic patterned surfaces as multifunctional liquid manipulators. ACS applied materials \& interfaces, 2017. 9(12): p. 10371-10377.

7. Lee, C.-S., et al., Protein patterning on silicon-based surface using background hydrophobic thin film. Biosensors and Bioelectronics, 2003. 18(4): p. 437-444.

8. Clancy, K.F., et al., Protein microarray spots are modulated by patterning method, surface chemistry and processing conditions. Biosensors and Bioelectronics, 2019. 130: p. 397-407.

9. Kuo, C.T., et al., ParaStamp and Its Applications to Cell Patterning, Drug Synergy Screening, and Rewritable Devices for Droplet Storage. Advanced biosystems, 2017. 1(5): p. 1700048.

10. Notsu, H., et al., Super-hydrophobic/super-hydrophilic patterning of gold surfaces by photocatalytic lithography. Journal of Materials Chemistry, 2005. 15(15): p. 1523-1527.

11. Kim, S.C., D.J. Sukovich, and A.R. Abate, Patterning microfluidic device wettability with spatiallycontrolled plasma oxidation. Lab on a Chip, 2015. 15(15): p. 3163-3169.

12. Rashid, Z., et al., Reversible switching of wetting properties and erasable patterning of polymer surfaces using plasma oxidation and thermal treatment. Applied Surface Science, 2018. 441: p. 841-852.

13. Yang, X., et al., A twice electrochemical-etching method to fabricate superhydrophobicsuperhydrophilic patterns for biomimetic fog harvest. Scientific reports, 2017. 7(1): p. 1-12.

14. Kim, H., et al., Drop impact characteristics and structure effects of hydrophobic surfaces with micro-and/ornanoscaled structures. Langmuir, 2012. 28(30): p. 11250-11257.

15. Gorman, C.B., H.A. Biebuyck, and G.M. Whitesides, Use of a patterned self-assembled monolayer to control the formation of a liquid resist pattern on a gold surface. Chemistry of materials, 1995. 7(2): p. 252-254.

16. Rios-Carvajal, T., et al., Specific ion effects on the interaction of hydrophobic and hydrophilic selfassembled monolayers. Langmuir, 2018. 34(35): p. 10254-10261.

17. Lee, S.H., et al., Multifunctional self-assembled monolayers via microcontact printing and degasdriven flow guided patterning. Scientific reports, 2018. 8(1): p. 1-8.

18. Liu, Q., et al., A polydopamine patterned perfluoropolymer-basedsubstrate for protein microarray applications. Sensors and Actuators B: Chemical, 2019. 287: p. 306-311.

19. Tse, C.C.W., et al., Utilising inkjet printed paraffin wax for cell patterning applications. 2016.

20. Slodowicz, M.P., et al., Physicochemical properties and field evaluation of monolithic wax formulations for the controlled release of a forest pest pheromone. Journal of the Brazilian Chemical Society, 2017. 28(2): p. 348-359. 
21. Carlen, E.T. and C.H. Mastrangelo, Surface micromachined paraffin-actuated microvalve. Journal of Microelectromechanical Systems, 2002. 11(5): p. 408-420.

22. Carlen, E. and C. Mastrangelo. Simple, high actuation power, thermally activated paraffin microactuator. in Transducers' 99 Conference, Sendai, Japan, June. 1999.

23. Jing, G., et al., Development of a microfluidic biosensorfor detection of environmental mycobacteria. Sensors and Actuators B: Chemical, 2007. 123(1): p. 614-621.

24. Jing, G., et al., Paraffin surfaces forculture-based detection of mycobacteria in environmental samples. Journal of Micromechanics and Microengineering, 2004. 15(2): p. 270.

25. Renault, C., et al., Three-dimensional wax patterning of paperfluidic devices. Langmuir, 2014. 30(23): p. 7030-7036.

26. Ren, K., et al., Convenient method for modifying poly (dimethylsiloxane) to be airtight and resistive against absorption of small molecules. Analytical chemistry, 2010. 82(14): p. 5965-5971.

27. Lee, J.N., C. Park, and G.M. Whitesides, Solvent compatibility of poly (dimethylsiloxane)-based microfluidic devices. Analytical chemistry, 2003. 75(23): p. 6544-6554.

28. Cai, Z., et al., Fluid separation and network deformation in wetting of soft and swollen surfaces. Communications Materials, 2021. 2(1): p. 1-11.

29. Dangla, R., F. Gallaire, and C.N. Baroud, Microchannel deformations due to solvent-induced PDMS swelling. Lab on a Chip, 2010. 10(21): p. 2972-2978.

30. Park, J.Y., et al., PDMS-paraffin/graphene laminated films with electrothermally switchable haze. Carbon, 2016. 96: p. 805-811.

31. Yao, X., et al., Temperature-Driven Switching of Water Adhesion on Organogel Surface. Advanced Materials, 2014. 26(12): p. 1895-1900.

32. Feng, W., E. Ueda, and P.A. Levkin, Droplet Microarrays: From Surface Patterning to HighThroughput Applications. Advanced materials, 2018. 30(20): p. 1706111.

33. $\mathrm{Xu}, \mathrm{K}$., et al., Self-partitioned droplet array on laser-patterned superhydrophilic glass surface for wall-less cell arrays. Analytical chemistry, 2016. 88(5): p. 2652-2658.

34. Walsh, E.J., et al., Microfluidics with fluid walls. Nature communications, 2017.8(1): p. 1-9.

35. Soitu, C., et al., Jet-Printing Microfluidic Devices on Demand. Advanced Science, 2020. 7(23): p. 2001854.

36. Milne, A. and A. Amirfazli, The Cassie equation: How it is meant to be used. Advances in colloid and interface science, 2012. 170(1): p. 48-55. 